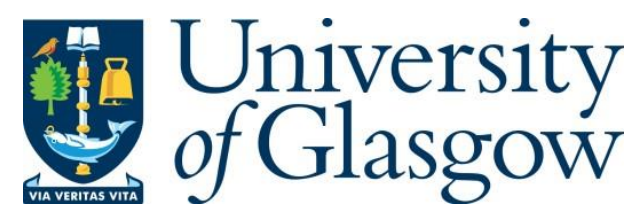

Chen, Y., Ghannam, R. and Heidari, H. (2019) Air Quality Monitoring Using Portable Multi-Sensory Module for Neurological Disease Prevention. In: 4th International Conference on UK - China Emerging Technologies (UCET 2019), Glasgow, UK, 21-22 Aug 2019, ISBN 9781728127972.

There may be differences between this version and the published version. You are advised to consult the publisher's version if you wish to cite from it.

http://eprints.gla.ac.uk/190522/

Deposited on: 23 July 2019

Enlighten - Research publications by members of the University of Glasgow http://eprints.gla.ac.uk 


\title{
Air Quality Monitoring using Portable Multi-Sensory Module for Neurological Disease Prevention
}

\author{
Yanjun Chen ${ }^{1,2}$, Rami Ghannam ${ }^{1}$ and Hadi Heidari ${ }^{1}$ \\ ${ }^{1}$ Microelectronics Lab (meLAB), School of Engineering, University of Glasgow, G12 8QQ, UK \\ ${ }^{2}$ University of Electronic Science and Technology of China, Chengdu, China \\ hadi.heidari@glasgow.ac.uk
}

\begin{abstract}
Improved standards of living and economic development have resulted in increased environmental and health problems. Due to the deterioration of air quality, the haze weather phenomenon is becoming a serious issue. It has been found that there may be a potential association or causal relationship between air pollution exposure and neurological diseases. In many geographical areas, haze weather phenomenon is incorporated into fog as a severe weather warning. This paper presents the design and implementation of a portable multi-sensory system for air pollution monitoring. This system consists of a microprocessor control unit as the control core, as well as temperature, humidity, gas and dust sensors. Moreover, an acoustooptic alarm system is included, which enables real-time collection, processing, display, and functions of temperature, humidity, harmful gases and particulate matter.
\end{abstract}

Keywords- Neurological Diseases, Particulate Matter Detection, Air Quality Monitoring.

\section{INTRODUCTION}

Air pollutants are caused by a mixture of gaseous, volatile substances, semi-volatile and particulate matter (PM) substances. The emission of these pollutants into the atmosphere are all man-made causes. Air pollution can lead to a series of health problems such as respiratory diseases [1], premature births [2], as well as related extended problems, which seriously threaten the safety of human life. The degree of damage to the neural system caused by air pollution ranks second among other health effects, only to the respiratory system [1]. There is growing evidence that air pollutants can not only harm the central neural system, accelerate cognitive aging, but may even increase the risk and mortality of Alzheimer's disease (AD), Parkinson's disease (PD) and other neurological diseases [3-6]. Cognitive impairment is an important clinical symptom in the early stage of neurologic diseases. Previous studies have explored the relationship between long-term exposure to air pollutants and cognitive impairment in the elderly [7].

At present, it is believed that the main risk factors of neurological diseases are genetic, age and environmental factors. Environmental exposure includes air exposure. Air pollution is a common environmental exposure factor. Long- term exposure to toxic substances in the air can cause gene mutations [5], which is also the culprit for the rejuvenation of neurological diseases. Both the causes of neurological diseases and the degrees of factors are different, which lead to different degrees of illness [4]. The pathway of how air pollution leads to the neurological disease is shown in Fig. 1.

The volatile organic pollutants (VOCs), which have photochemical activity, are important components of compound air pollution [8], and PM2.5 is the particulate

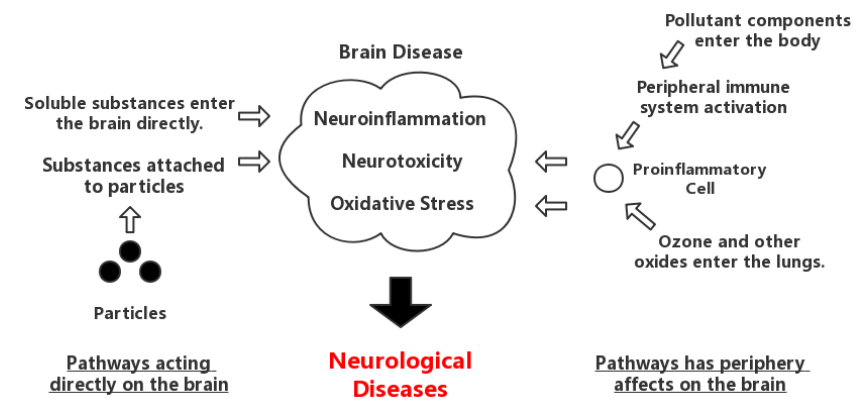

Fig. 1. Pathway of air pollution affects neurological disease.

matter published after PM10. PM2.5 is more susceptible to toxic and harmful substances, and stays in the atmosphere for a longer time. Thus, it has a greater impact on human health and the quality of the atmospheric environment [9]. In the modern monitoring system, extraction technology and chromatographic technique [8] for detecting VOCs, $\beta$-ray method and oscillatory balance method [10] for monitoring PM2.5 are widely used in commercial machines and factories. Machines are usually cumbersome, so they can't be carried around. They are expensive so cannot be widely used in everyday life. Compared with the traditional method, the system using sensors to monitor air quality is more efficient, simple in circuit design and has wide applicability [11-15].

In this paper, a portable sensory system for air pollution monitoring is introduced, and the block diagram has been shown in Fig. 2. It can measure, monitor and display the temperature and humidity, harmful gas and PM2.5 through multiple sensors, keys, a screen and other modules. The collected data is displayed on the screen after MCU processing. The accuracy of the measurements is comparable 


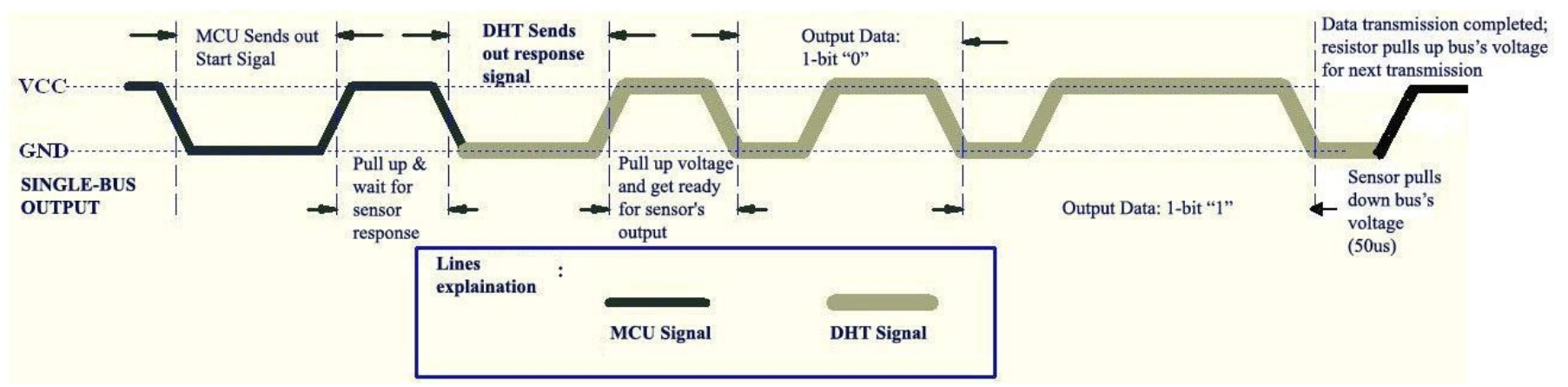

Fig. 3. Time series of DHT11.

to that used by the expensive and large commercial air pollution prediction system. When the measured value exceeds the safety range, the system will send out a corresponding optical and sound alarm signal, which represents a threat to human health.

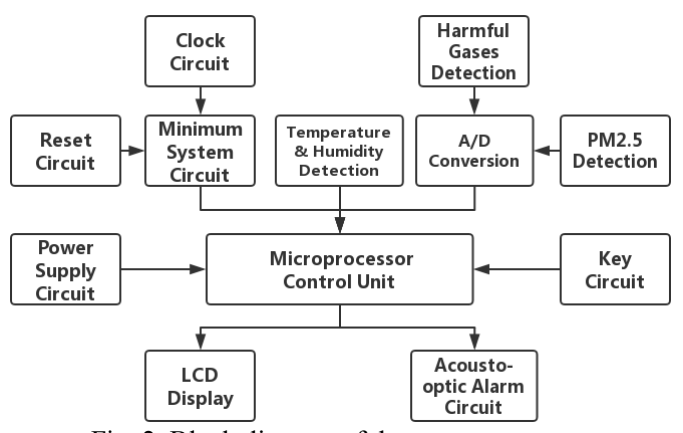

Fig. 2. Block diagram of the sensory system.

This product has a compact size, low power consumption, simple operation and is suitable for indoor placement and carry out. It can help people know air quality in real time, so as to ensure environmental and neurological safety.

\section{CONSTITUENT STRUCTURE}

STC89C52RC single chip microcomputer is an 8-bit highperformance micro-control processor which is often used at present. It uses the centralized control mode of special function register to control all kinds of functional components with low price, well-developed technology, simple operation and other characteristic. It provides different operating modes and power-saving modes to save power. Meanwhile, it has a single-chip microcomputer minimum system composed of crystal oscillator circuit and reset circuit [16]. This design adopts the crystal oscillator of $11.0592 \mathrm{MHz}$ and adopts the combination of automatic power-on and key reset.

DHT11 is selected as a designed sensor for temperature and humidity detection. It is a digital sensor that combines temperature and humidity measurement, which uses digital module acquisition technology and sensing technology to ensure that the product has extremely high reliability and excellent long-term stability. It communicates with the singlechip microcomputer through a single bus, so it takes up less resource. The specific format for transmitting data is the sum of 8-bit integer / decimal data of temperature / humidity and the 8-bit checksum. That is, when the data is transferred correctly, the checksum data is equal to the last 8 bits of the result of the four 8-bit data above. The sensor is controlled with time series, which leads to work stably and accurately [17]. The control process for the specific time series is presented in the following Fig. 3.

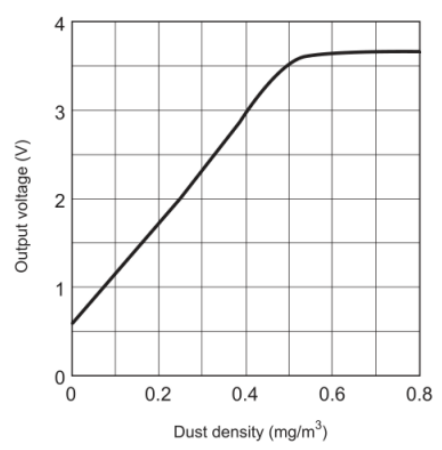

Fig. 4. Output voltage vs Dust density.

Dust sensor GP2Y1014AU is selected in this product for low power consumption and high stability. In the sensor, an infrared light-emitting diode and a phototransistor are installed diagonally, and a hole in the centre of the sensor allows the air to flow freely. When the LED emits light, the reflected and refracted light from the particles in the air can be detected and used to determine the size and content of the particles. The size and quantity of different particles output analog voltage values of different sizes, and which is proportional to the concentration of dust [18]. The basic relationship of the scale is shown in the following Fig. 4. Depending on the driving period and the driving time, that is the pulse period and pulse width, or the change of the power supply voltage, the output voltage will change [18].

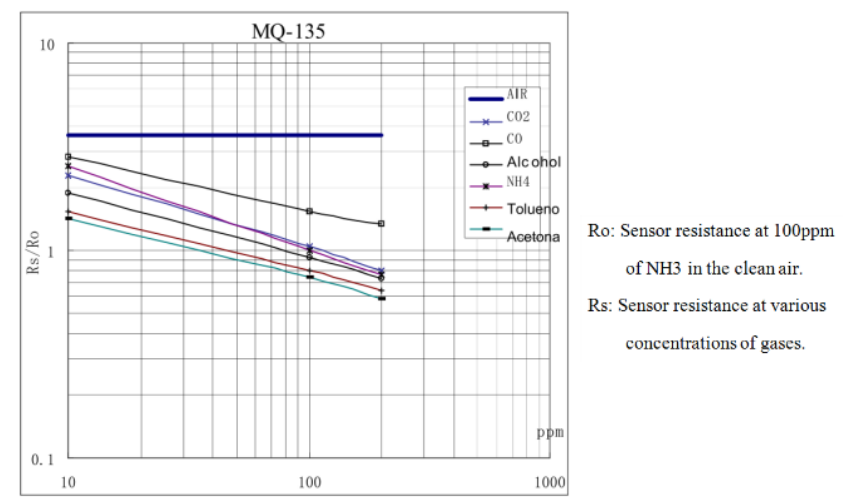

Fig. 5. Typical sensitivity characteristics of MQ-135 for several gases. 
MQ-135 has a certain long-term stability, sensitivity response recovery characteristics and sensitivity characteristics, in this system is mainly used to detect the concentration of benzene, nitrogen oxides, ammonia and other harmful gases in the air. The gas sensing material used is tin dioxide $(\mathrm{SnO} 2)$, which has good sensitivity to harmful gases in a wide range of concentrations. In clean air, the conductivity of the sensor is low. When there are polluted gases in the environment of the sensor, the conductivity of the sensor increases with the increase of the concentration of the polluted gases in the air. For different gases, the typical sensitivity characteristics of the sensor will be different [19]. The specific properties curve is shown in the following Fig. 5.

Both of these two sensors need to be connected to the converter $\mathrm{ADC} 0832$ for $\mathrm{A} / \mathrm{D}$ conversion, to turn non-electric analog signals into corresponding analog signals, and then convert analog signals into corresponding digital signals, so that the analog signals can be processed by software.. ADC0832 has dual data output which can be used as data verification to reduce the error of data, and the conversion speed is fast and the stability is strong [20].

LCD1602 is a commonly used liquid crystal display, can display numbers, symbols, graphics, and so on, which is widely used in a variety of fields. It has high quality and will not flash. And single-chip computer system interface is more simple and reliable, which is more convenient for operation [21]. In this system, it is selected to combine with the key module to display the measured values of the sensors and the values set by the keys. The principle of display is to make use of the physical characteristics of liquid crystal to control the display area through voltage [21].

Simultaneously, only a few function keys are needed in the control system, so the independent key structure of the noncoding keyboard is adopted, which is flexible and the software structure is simple. The level state of the I/O port can be used to determine the key action, thereby executing the set instructions. A total of four keys are selected, one of which sets the key to function as a mode switch, a plus key, a subtractive key, and a key controls the switch on and off of the buzzer. The software method is used to eliminate the jitter, the process is to continuously detect the key value, until the key value is stable [22].

The acousto-optic alarm system consists of four red light emitting diodes and an active buzzer. When the temperature exceeds the maximum or below the minimum setting value, the LED1 light is on. When the humidity exceeds the maximum or below the minimum setting value, the LED2 light is on. When the dust concentration is higher than the maximum set value, the LED3 is on. When the concentration of harmful gases is higher than the maximum set value, the LED4 is on. The air quality is low when the LED3 or LED4 is on. The product is introduced in Fig. 6.

The control core of the software system is the main program of the system, which completes the control of each part by calling each sub-program. The main program forms a while loop, each call to a subroutine that is to perform a part of the function. After the single-chip computer is powered on,

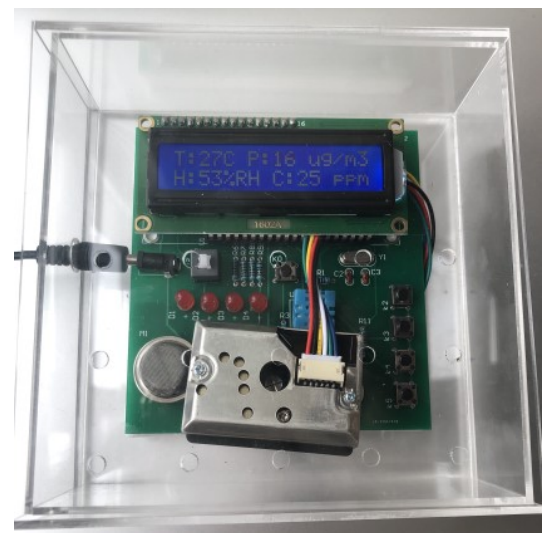

Fig. 6. The portable sensory box for monitoring of air pollution.

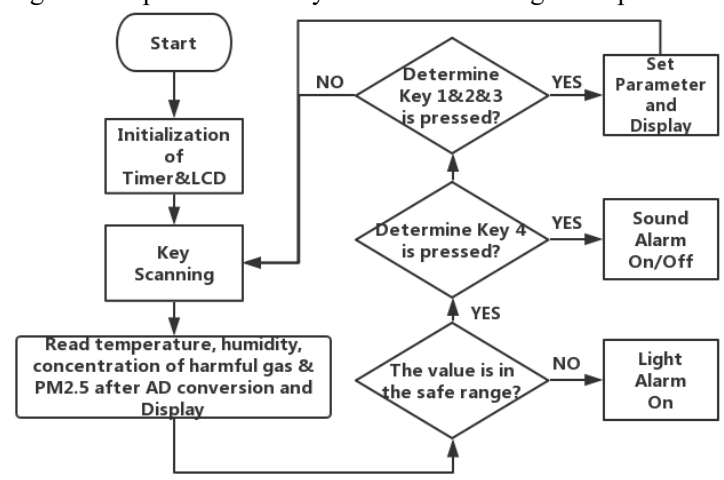

Fig. 7. Block diagram of the program.

the main program is executed first from the block diagram in Fig. 7, the initial subroutine of the system is executed first in the main program. Once all initialization have been executed, the process will enter a large loop of the system. In the loop, a number of environmental parameters detection, key scanning detection, alarm judgment and others will be executed. So far, once cycle of while cycle ends, and then a new round of detection, display, alarm will be carried out, unless the reset key is pressed, or the program runs away. The real-time detection of environmental parameters can be realized in the cycle and the alarm can be issued immediately after an environmental parameter value is found to exceed the limit at the first time.

\section{DESIGN AND FABRICATION}

In the process of air quality evaluation, the whole system needs to have good ventilation. For each component to function properly for a period of time, they should be stored in a stable space. Therefore, the printed circuit board is selected to complete the circuit design. To form a complete system, an outer package is needed. Given the existence of LCD display and keyboard in the system, the box must be designed to have a transparent and open-fit style in order to match the design better as a whole. As described above, acrylic sheet is selected, which is an important plastic polymer material developed earlier, with good transparency, chemical stability and weather resistance. Based on the size of PCB, the size of the outer packaging box is $120 * 120 * 45 \mathrm{~mm}$, the thickness of the sheet is $2 \mathrm{~mm}$. It is divided into two parts with a shell and an inner box included. The 3D model drawings are shown in Fig. 8. 


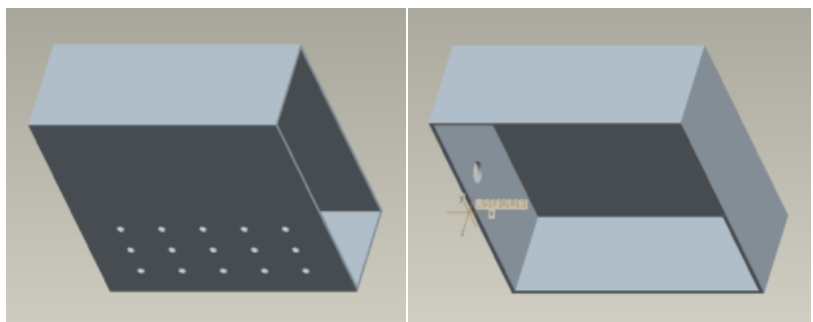

Fig. 8. 3D Model design of outer package.

IV. EXPERIMENTAL RESULTS

After the hardware circuit is built and the software program is written and debugged, the two are combined in a single system and loaded into the single-chip microcomputer. After experiments, it is found that the detection of temperature, humidity and harmful gas concentration can be measured accurately, while that of PM2.5 concentration sometimes change periodically, but the overall measurement results do not produce excessive errors. The key module and acoustooptic alarm system can correctly implement the settings and alarm work according to different conditions, and the LCD display screen can clearly display the data without shadow. All the subroutine of the software can work properly. Outer box and the whole system can also match perfectly, which will not have an adverse impact on the use of sensors. The results of the simulation output and the actual output are shown in Fig. 9.
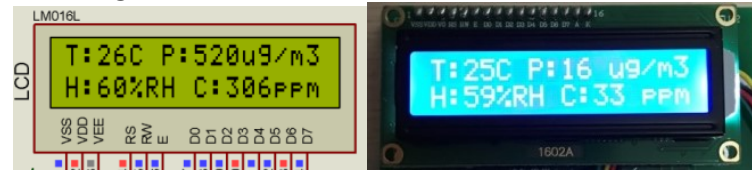

Fig. 9. The output of simulation and the actual output on LCD1602.

\section{CONCLUSIONS}

In this paper, a portable air pollution monitoring system has been designed and implemented, which has a high costperformance ratio and can be widely used in various environments. One of the advantages of this design is that it can simultaneously measure and display temperature, humidity, harmful gases, PM2.5 and four environmental parameters. In theory, the system can achieve 24-hour uninterrupted operation. In practice, due to a long power-up time, this may lead to the heating of the components, which will affect the accuracy of the detection system. Due to these limitations, the heat dissipation system needs further investigations. Furthermore, high-precision and high-quality sensors can be optimized within the system to improve the accuracy and generality of measurements.

\section{REFERENCES}

[1] Ox M. A., Tran N. L, Groopman J. D. et al., “Toxicological resources for cumulative risk : an example with hazardous air pollutants," Regul Toxicol Pharmacol, vol. 40, no. 3, pp. 305-311, 2004.

[2] Guo, Le Qian, et al., "Ambient air pollution and adverse birth outcomes: a systematic review and meta-analysis*\#," Journal of Zhejiang University (Series B for biomedicine and biotechnology), 2019.
[3] Yegambaram M, Manivannan B, Beach $T$ G et al., "Role of environmental contaminants in the etiology of Alzheimer's disease: a review," Curr Alzheimer Res, vol. 12, no. 3, pp. 116-146, 2015.

[4] Chen J C, Wang X, Wellenius G A, et al., "Ambient air pollution and neurotoxicity on brain structure: evidence from women's health initiative memory study," Ann Neurol, vol. 78, no. 3, pp. 466-476, 2015.

[5] Zanobetti A, Dominici F, Wang Y, Schwartz JD, "A national casecrossover analysis of the short-term effect of PM2.5 on hospitalizations and mortality in subjects with diabetes and neurological disorders," Environ Health, vol. 13, no. 1, pp. 38, May. 22, 2014.

[6] IOS Press, "Air pollution in Mexico City is associated with the development of Alzheimer disease: The evolving axonal damage can be identified using a novel Non-P-Tau assay for cerebrospinal fluid CSF," ScienceDaily, Dec. 21, 2018.

[7] Gatto NM, Henderson VW, Hodis HN, et al., "Conponents of air pollution and cognitive decline in older adults in Los Angeles," Neurotoxicology, vol. 40, pp.1-7, 2014.

[8] Ji Yihu, "Research on monitoring technology and treatment of volatile organic pollutants (VOCs)," Low-carbon World, 2018.

[9] Li Huijuan, Zhou Dequn and Wei Yongjie, "Impact Factors of Air Pollution in Chinese Important Cities: Based on Source Apportionment," Ecological Economy, 2018.

[10] Yang Liping, Li Dechun, and Liu Haijiang, "Comparison of PM2.5 automatic monitoring methods in ambient air and application analysis of monitoring instruments," Chinese High-tech, vol. 1, no. 17, pp. 6264, 2017.

[11] R. Parvizi, S. Azad, K. Dashtian, M. Ghaedi, and H. Heidari, "Natural Source-Based Graphene as Sensitising Agents for Air Quality Monitoring," Scientific Reports, vol. 9, p. 3798, 2019.

[12] V. Nabaei et al., "Magnetic Biosensors: Modelling and Simulation", Biosensors Bioelectronics, vol. 103, pp. 69-86, 2018.

[13] S. Samyak et al., "On-chip Magnetoresistive Sensors for Detection and Localization of Paramagnetic Particles", Proc. of IEEE SENSORS Conference, 2017.

[14] X. Liang, R. Ghannam and H. Heidari, "Wrist-Worn Gesture Sensing With Wearable Intelligence," in IEEE Sensors Journal, vol. 19, no. 3, pp. 1082-1090, 1 Feb.1, 2019.

[15] X. Zhao, S. Zuo, R. Ghannam, Q. H. Abbasi and H. Heidari, "Design and Implementation of Portable Sensory System for Air Pollution Monitoring Monitoring," 2018 IEEE Asia Pacific Conference on Postgraduate Research in Microelectronics and Electronics (PrimeAsia), Chengdu, 2018, pp. 47-50.

[16] Chen Danhua, "Design and Application of Single Chip Microcomputer Minimum System,“ Wireless interconnection technology, vol. 10, pp. 103-104, 2012.

[17] D-Robotics UK, DHT11 Humidity \& Temperature Sensor, 2010.

[18] SHARP Corporation, Data sheet for GP2Y1010AU0F sensor, 2006.

[19] HANWEI Electronics Co.,Ltd, Technical Data of MQ-135 Gas Sensor, 2014.

[20] Zhang Yalin, "Application details of ADC0832 based on C52 control," Digital technology and application, vol. 3, 2013

[21] .TINSHARP Corporation, TC1602B-01 VER:00 Specification For Approval, 2005

[22] Huang Jingqi, "Elimination of key jitter based on FPGA," Chinese high-tech enterprise, vol. 21, pp. 31-32, 2013. 